\title{
Estrogen receptor $\beta$ promotes bladder cancer growth and invasion via alteration of miR-92a/DAB2IP signals
}

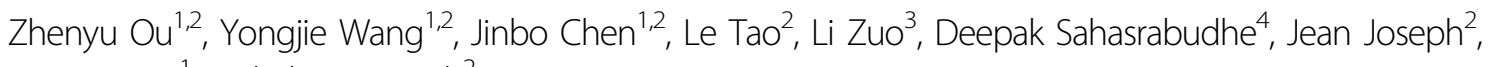
Long Wang ${ }^{1}$ and Shuyuan Yeh ${ }^{2}$

\begin{abstract}
Although early studies suggested that bladder cancer $(\mathrm{BCa})$ is more prevalent in men than in women, muscle-invasive rates are higher in women than in men, suggesting that sex hormones might play important roles in different stages of BCa progression. In this work, we found that estrogen receptor beta (ERB) could increase BCa cell proliferation and invasion via alteration of miR-92a-mediated DAB2IP (DOC-2/DAB2 interacting protein) signals and that blocking miR92a expression with an inhibitor could partially reverse ER $\beta$-enhanced BCa cell growth and invasion. Further mechanism dissection found that ER $\beta$ could increase miR-92a expression at the transcriptional level via binding to the estrogen-response-element (ERE) on the $5^{\prime}$ promoter region of its host gene C13orf25. The ER $\beta$ up-regulated miR-92a could decrease DAB2IP tumor suppressor expression via binding to the miR-92a binding site located on the DAB2IP $3^{\prime}$ UTR. Preclinical studies using an in vivo mouse model also confirmed that targeting this newly identified ERß/miR-92a/ DAB2IP signal pathway with small molecules could suppress BCa progression. Together, these results might aid in the development of new therapies via targeting of this ERß-mediated signal pathway to better suppress BCa progression.
\end{abstract}

\section{Introduction}

Bladder cancer $(\mathrm{BCa})$ is a urological malignancy that has the highest lifetime treatment cost per patient among all types of solid cancers ${ }^{1}$. The incidence of $\mathrm{BCa}$ is nearly 3fold higher in men than in women ${ }^{2}$. In contrast, the survival rate in female $\mathrm{BCa}$ patients is less than that in male $\mathrm{BCa}$ patients ${ }^{3}$. These results suggest that sex hormones, including androgen, androgen receptor, estrogens, and estrogen receptors (ERs), might contribute to this gender difference in $\mathrm{BCa}$ progression. The two major types of ERs are ER-alpha (ER $\alpha)$ and ER-beta $(E R \beta)$. Results from gene knockout mouse models showed that ER $\alpha$ has a protective

\footnotetext{
Correspondence: Long Wang (doctorwanglong@163.com) or Shuyuan Yeh (shuyuan_yeh@urmc.rochester.edu)

${ }^{1}$ Departments of Urology and Plastic Surgery, Xiangya Hospital, Central South University, Changsha 410008, China

${ }^{2}$ Departments of Urology and Pathology, University of Rochester Medical

Center, Rochester, New York 14642, USA

Full list of author information is available at the end of the article.

These authors contributed equally: Zhenyu Ou and Yongjie Wang
}

role in inhibiting $\mathrm{BCa}$ initiation and growth, and $\mathrm{ER} \beta$ promotes $\mathrm{BCa}$ cell growth and invasion ${ }^{4,5}$. However, the underlying mechanisms by which $\mathrm{ER} \beta$ promotes $\mathrm{BCa}$ progression remain largely unclear. The microRNAs (miRNAs, miRs) are small highly conserved noncoding RNAs that can post-transcriptionally regulate target genes via binding to the $3^{\prime}$ untranslated region (3'UTR) of mRNAs ${ }^{6}$. Accumulating evidence indicates that miRNAs play important roles in tumor progression and in $\mathrm{BCa}$ growth and invasion ${ }^{7,8}$. The miRNAs can act as oncogenes to promote tumor development or as tumor suppressors to inhibit cancer development ${ }^{9}$. ER $\beta$ has been reported to directly regulate several miRNAs and affect tumor progression in different organs or tumors ${ }^{10}$. However, whether $\mathrm{ER} \beta$ can promote $\mathrm{BCa}$ via regulation of miRNAs has not been fully investigated.

DOC-2/DAB-2 interacting protein (DAB2IP) belongs to the Ras GTPase-activating protein family and functions as a tumor suppressor to mediate tumor growth and 
invasion ${ }^{11}$. Clinical data indicated that DAB2IP was down-regulated in different human cancers, including prostate, lung, liver, and bladder ${ }^{12-15}$. In $\mathrm{BCa}$, a low expression of DAB2IP was found to be associated with aggressive clinical features and worse outcomes ${ }^{16}$.

In this work, we introduce a previously unexplored mechanism by which ER $\beta$ modulates miR-92a/DAB2IP signals to promote $\mathrm{BCa}$ cell growth and invasion.

\section{Materials and methods}

\section{Cell lines}

Human BCa cell lines UMUC3 and J82 were purchased from the American type culture collection (ATCC, Ma-nassas, VA) and cultured in Dulbecco's modified eagle media (DMEM) supplemented with 10\% FBS, $2 \mathrm{mM} \mathrm{L}$-glutamine, $100 \mathrm{IU} / \mathrm{mL}$ penicillin, $50 \mu \mathrm{g} / \mathrm{mL}$ streptomycin and maintained in a humidified $5 \% \mathrm{CO}_{2}$ environment at $37^{\circ} \mathrm{C}$.

\section{Cell growth assay}

Different $\mathrm{BCa}$ cells $\left(\right.$ at $5 \times 10^{3}$ ) were plated into each well of 24-well plates. Viable cells were quantified at days $0,2,4$, and 6 by incubation of cells in $0.5 \mathrm{mg} / \mathrm{ml}$ of 3-(4,5dimethylthiazol-2-yl)-2,5-diphenyltetrazolium bromide (MTT) (Sigma-Aldrich, St. Louis, MO, USA) for $1 \mathrm{~h}$ and dissolution with DMSO. The absorbance was measured at a wavelength of $570 \mathrm{~nm}$ and data were presented as relative changes (fold).

\section{Transwell invasion assays}

Invasion assays were performed using transwells with 8 $\mu \mathrm{m}$ pore-size inserts (Corning Inc., Corning, NY). The upper chamber inserts were coated with diluted Matrigel (BD Biosciences, Sparks, MD) for invasion assays. Amounts of $5 \times 10^{4} \mathrm{BCa}$ cells (in serum-free media) and $10 \%$ serumcontaining media were plated in the upper and lower chambers, respectively. After a 24-hr incubation, the cells that invaded to the bottom sides of the transwell membranes were fixed and stained with $0.1 \%$ crystal violet. Positively stained cells were counted from six random fields. Quantitation is expressed as mean \pm SD of triplicate repeats.

\section{D invasion assay}

The 3D invasion assay was conducted according to a previous study ${ }^{17}$. In brief, Matrigel was thawed on ice, added to each well of 8-well glass chamber slides (at 50 $\mu \mathrm{l} / \mathrm{cm}^{2}$ ) and spread evenly. Amounts of $1 \times 10^{5} \mathrm{~J} 82$ cells were placed onto each well. Approximately 10 days later, the $\mathrm{BCa}$ cells were observed to form acini-like structures.

\section{RNA extraction and real-time quantitative PCR (q-PCR) analysis}

Total RNAs were isolated using TRIzol reagent (Invitrogen, Grand Island, NY), and $2 \mu \mathrm{g}$ was applied for reverse transcription using Superscript III transcriptase
(Invitrogen). Quantitative real-time PCR (qRT-PCR) was conducted using a Bio-Rad CFX96 system with SYBR green to measure the mRNA expression level of the gene of interest. Expression levels were normalized to the expression of GAPDH mRNA.

\section{Western blot analysis}

Western blot analysis was performed as previously described $^{4,5}$. In brief, cells were washed with PBS and lysed in RIPA buffer. Quantified proteins were separated using $10 \%$ sodium dodecyl sulfate polyacrylamide gel electrophoresis and transferred onto PVDF membranes. After blocking with non-fat milk, the membranes were incubated with appropriate dilutions of specific primary antibodies. After washing in Tris-buffered saline plus $0.05 \%$ Tween-20, the blots were incubated with peroxidase-conjugated secondary antibodies and visualized using an enhanced chemiluminescence system (Thermo Fisher Scientific, Waltham, MA).

\section{Lentivirus packaging and transfection}

$E R \beta$ shRNA was cloned into the lentiviral vector pLKO.1. To express ER $\beta$, the cDNA of ER $\beta$ was cloned into the pWPI vector. Subsequently, $293 \mathrm{~T}$ cells were transfected with lentiviral constructs, packaging, and envelope plasmids (psPAX2 and pMD2.G) to produce the lentivirus. After $48 \mathrm{~h}$, the lentivirus soup was harvested for immediate use or frozen for later use. The collected viruses were added to transduce the target cells for $24 \mathrm{hr}$. Cell cultures were refreshed with culture media and cultured for another three days to allow change of target protein expression.

\section{DAB2IP 3'UTR luciferase reporter assay}

Promoters of the miR-92a host gene were obtained from genomic DNA analysis of HEK293T cells using Phusion High-Fidelity DNA Polymerase (NEB, Ipswich, MA) and constructed into a pGL3-basic vector (Promega, Madison, WI) using the Gibson assembly method. Fragments of DAB2IP 3'UTR containing wild-type or mutant miRNAbinding sites were cloned into the psiCheck2 construct (Promega) downstream of the Renilla luciferase ORF. Cells were plated in 24-well plates and transfected with cDNA using Lipofectamine 3000 (Invitrogen). The thymidine kinase promoter-Renilla luciferase reporter plasmid (pRL-TK) was used as the transfection efficiency control. Luciferase activity was measured via the DualLuciferase Assay (Promega) according to the manufacturer's manual.

\section{Immunohistochemistry}

Mouse bladder tissues were fixed in 10\% (vol/vol) natural buffered formalin and embedded in paraffin. The embedded tissues were cut into sections with $5 \mu \mathrm{m}$ 
thickness. The tissue sections were deparaffinized in xylene solution and rehydrated using gradient ethanol concentrations. Immunostaining was performed as described previously ${ }^{17}$.

\section{In vivo mouse $\mathrm{BCa}$ studies}

Female nude mice 6-7 weeks of age were purchased from NCI. J82 cells were stably transfected with luciferase reporter gene (pcDNA3.0-luciferase) for monitoring of tumor growth and metastasis via the real-time in vivo imaging system (IVIS). Orthotopic xenografts of these $\mathrm{BCa}$ cells were applied according to our previous study $^{17}$. In brief, after the female mice (7-8 weeks of age) were anesthetized, a lower mid-line abdominal incision was made, and the bladder was exposed. Amounts of $1 \times 10^{6} \mathrm{~J} 82$ cells of different groups were resuspended in PBS and mixed with Matrigel (1:1). The cells were orthotopically injected into the muscle layer of bladder wall. Five weeks after tumor cell implantation, mice were injected with $150 \mathrm{mg} / \mathrm{kg}$ luciferin, and the fluorescent Imager (IVIS Spectrum, Caliper Life Sciences, Hopkinton, MA) was applied to monitor tumor growth and metastasis in live mice weekly. After eight weeks of tumor cell implantation, the mice were sacrificed, and the primary and metastatic tumors were further examined.

\section{Statistical analysis}

All data are presented as the mean \pm SD from at least three independent experiments. Statistical analyses were performed with SPSS 17.0 (SPSS Inc., Chicago, IL). Differences between two groups were analyzed using the unpaired Student's $t$-test. $P<0.05$ was considered statistically significant.

\section{Results}

\section{$E R \beta$ increases $B C a$ cell proliferation and invasion}

To test the ER $\beta$ impacts on $B C a$ cell growth and invasion, we first transfected ER $\beta$-cDNA or ER $\beta$-shRNA into BCa UMUC3 and 82 cells, respectively, using the lentivirus system (Fig. 1a) and applied the MTT growth assay to study their effects. The results revealed that knockdown of ER $\beta$ with ER $\beta$-shRNA in BCa J82 cells could decrease $B C$ a cell growth, and ectopic expression of ER $\beta$ with ER $\beta$-cDNA in UMUC3 cells could increase BCa cell growth, suggesting that ER $\beta$ might promote $\mathrm{BCa}$ cell growth (Fig. 1b).

To further examine the ER $\beta$ effects on BCa cell invasion, we applied the Matrigel invasion assay, and the results revealed that knockdown of ER $\beta$ in J82 cells decreased $\mathrm{BCa}$ cell invasion and that overexpression of $\mathrm{ER} \beta$ in $\mathrm{BCa}$ UMUC3 cells increased $\mathrm{BCa}$ cell invasion (Fig. 1c). Similar results were also obtained when we replaced the Matrigel invasion assay with a 3D invasion assay showing that knockdown of ER $\beta$ in J82 cells significantly decreased cell invasion (Fig. 1d).

Taken together, the results from experiments using different $\mathrm{BCa}$ cell lines and assays all demonstrate that $\mathrm{ER} \beta$ can increase $\mathrm{BCa}$ cell proliferation and invasion.

\section{$E R \beta$ increases $B C a$ cell proliferation and invasion via an increased miR-92a expression}

To dissect the molecular mechanism of how ER $\beta$ increases $\mathrm{BCa}$ cell proliferation and invasion, we focused on miRNAs as a growing body of evidence indicates that miRNAs could play important roles in tumor progres$\operatorname{sion}^{18,19}$. We applied the qRT-PCR assay to screen $30 \mathrm{BCa}$ progression-related miRNAs after transduction of lentiviral ER $\beta$-shRNA (shER $\beta$ ) in J82 cells or ER $\beta$-cDNA in UMUC3 cells. The results revealed that the expression of miR-92a and miR-129 were the most selectively altered after modulation of ER $\beta$ (Fig. 1e).

We examined the miR-92a and miR-129 effects on BCa cell invasion, and the results revealed that transduction of BCa with lentiviral miR-92a, but not miR-129, increased $\mathrm{BCa}$ cell invasion (Fig. 1f). Importantly, the results from The Cancer Genome Atlas (TCGA) databases further revealed that $\mathrm{BCa}$ has higher miR-92a expression than normal bladder tissues (Fig. 1g), suggesting miR-92a might play positive roles in promotion of $\mathrm{BCa}$ progression.

We examined the miR-92a effects on the ER $\beta$-regulated $\mathrm{BCa}$ progression. We altered the expression of ER $\beta$ and miR-92a in J82 and UMUC3 cells (Fig. 2a), and examined the miR-92a effects on the ER $\beta$ influences on BCa progression. The results revealed that knockdown of ER $\beta$ in J82 cells decreased J82 cell growth and invasion and that further ectopic expression of miR-92a could partially reverse ER $\beta$-shRNA-inhibited $\mathrm{BCa}$ cell growth and invasion (Fig. 2b, d). In an opposite approach, we also found that addition of ER $\beta$ in UMUC3 cells (UMUC3-oeER $\beta$ ) led to promote the UMUC3 cell growth and invasion, and then transfection with miR-92a inhibitor (oligonucleotide) can partially reverse oeER $\beta$-increased cell growth and invasion in UMUC3 cells (Fig. 2c, e).

Taken together, the results (Fig. 1e-g and Fig. 2a-e) from $2 \mathrm{BCa}$ cell lines using different approaches all suggest that ER $\beta$ could increase $\mathrm{BCa}$ cell proliferation and invasion via alteration of miR-92a expression.

\section{Mechanism dissection: The ER $\beta$-upregulated miR-92a can promote $B C a$ cell growth and invasion via down-regulation of DAB2IP}

To dissect the molecular mechanism(s) of how ER $\beta$ upregulated miR-92a can promote $\mathrm{BCa}$ cell growth and invasion, we analyzed the miRNA prediction databases for its potential downstream target genes and identified the DAB2IP tumor suppressor as a potential $\operatorname{target}^{20}$. We 


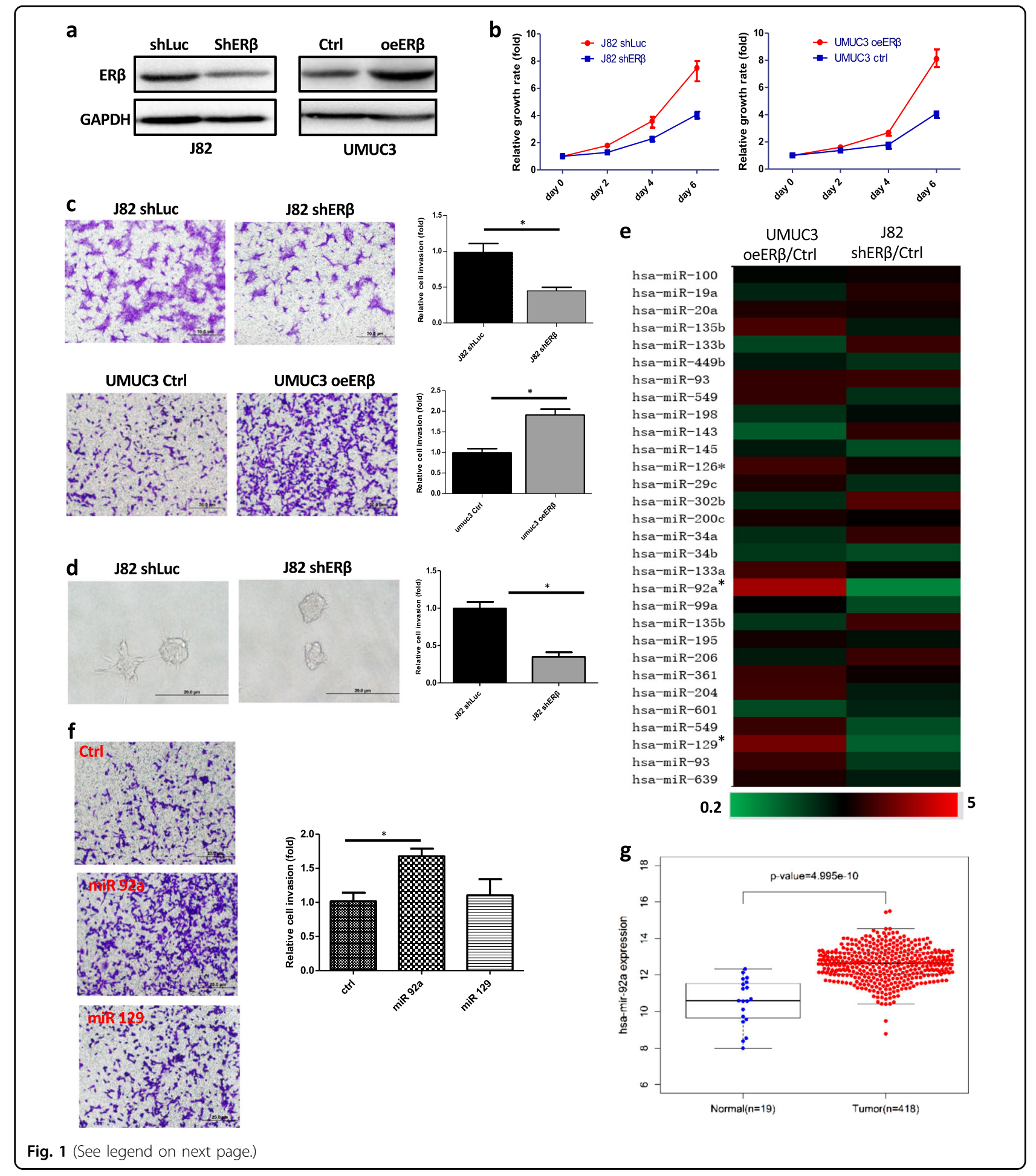

applied Western blot analysis to confirm the protein expression and found that the addition of miR-92a could significantly decrease DAB2IP expression and that transfection of BCa cells with miR-92a inhibitor could increase DAB2IP expression (Fig. 3a). Importantly, a human clinical survey using the cBioPortal database ${ }^{21}$ also identified a negative correlation between the expressions of ER $\beta$ and DAB2IP (Fig. 3b).

We next applied the interruption approach to test the functional connections among ER $\beta$, miR-92a, and DAP2IP in J82 and UMUC3 cells. The results with J82 cells revealed that knockdown of ER $\beta$ with ER $\beta$-shRNA 
(see figure on previous page)

Fig. 1 Identification of miRNA-92a as the downstream effector of ER $\beta$ in BCa. UMUC3 cells were transduced by lentivirus to overexpress $E R \beta$ (oeERß) or vector control (Crtl). J82 cells were transduced with lentiviral-shER $\beta$ to knock down ERß, or -shLuc as control for the following experiments. a Cell lysates were collected from lentivirus-transduced J82 and UMUC3 cells. Western blot analysis was performed, and proteins were detected by antibody against ERß. b The MTT assay was used to analyze cell growth in J82 and UMUC3 cells transduced as described above to overexpress or knock down ERß. c The Matrigel invasion assays were performed in $J 82$ and UMUC3 cells transduced as described above. $\mathbf{d}$ 3D invasion assay showed that more protruding structures formed in control J82 cells than in J82 shER $\beta$ cells. e Real-time PCR screening of ERß-regulated miRNAs related to BCa progression. We compared the PCR profile of miRNAs related to cancer cell growth and invasion in UMUC3 cells with overexpressed ER $\beta$ vs. vector (Ctrl) and J82 cells with shER $\beta$ vs. shLuc control (Ctrl). f Among the miRNAs screened, miR-92a and miR-129 expression were the most up-regulated by ERR. We transduced lentiviral-miR-92a and lentiviral miR-129 into BCa cells, and the results showed that the increased miR-92a, not miR-129, can selectively increase BCa invasion. $\mathbf{g}$ We used the TCGA database to analyze the BCa sample array with miR-92a expression, and the results showed that BCa tumors have higher miR-92a expression than normal bladder tissues. In $\mathbf{c}$, $\mathbf{d}$, and $\mathbf{f}$, data are presented as the mean \pm SD. ${ }^{*} p<0.05$

$(\operatorname{shER} \beta)$ could increase DAB2IP expression and reduce J82 cell invasion (Fig. 3c, lanes 1 vs 2). Targeting of tumor suppressor DAB2IP with shRNA (shDAP2IP) can reduce the shER $\beta$-increased DAP2IP protein (Fig. 3c, lanes 4 vs. 3, upper panel). Importantly, the addition of lentiviral shDAB2IP can effectively reverse the shER $\beta$-reduced J82 cell invasion (Fig. 3c, lanes 2 vs 4, lower panel). As UMUC3 cells have a lower endogenous ER $\beta$ expression, we over-expression of $E R \beta$ (oeER $\beta$ ) to examine the ER $\beta$ effects on the DAB2IP protein expression and UMUC3 cell invasion. Results showed that oeER $\beta$ can downregulated DAB2IP and increase UMUC cell invasion (Fig 3d, lanes 1 vs 2, lower panel). Furthermore, the ectopic DAP2IP over-expression (oeDAP2IP) can reverse the oeER $\beta$ up-regulated UMUC cell invasion (Fig. 3d, lanes 2 vs 4 , lower panel). As the oeDAP2IP can significantly increase the protein expression, the changes of endogenous DAP2IP protein bands in lanes 1 and 2 were barely observed due to the relative detection of protein amount difference (Fig. 3d, upper panel). Indeed, the oeER $\beta$-down-regulated DAP2IP can be better observed in UMUC3 in the Fig. 3e (lanes 1 vs 2, right panel). Supportively, we found that ER $\beta$-shRNA-increased DAB2IP expression could be significantly reversed after addition of miR-92a to J82 cells (Fig. 3e, left panel). As expected, oeER $\beta$-decreased DAB2IP expression (Fig. 3e, right panel) could also be effectively reversed after transfection of UMUC3 cells with miR-92a inhibitor.

Taken together, the results from clinical $\mathrm{BCa}$ tissues and 2 different $\mathrm{BCa}$ cell studies (Fig. 3a-e) consistently show that a functional connection exists among ER $\beta$, miR-92a, and DAB2IP. The ER $\beta$ could up-regulate oncogenic miR-92a to down-regulate the tumor suppressor DAB2IP, and then increase $\mathrm{BCa}$ cell invasion.

\section{Mechanism dissection: ER $\beta / m i R-92 a$ can modulate DAB2IP expression via binding to the DAB2IP 3 '-UTR}

To further dissect the molecular mechanism by which miR-92a regulates DAB2IP expression, we tested whether miR-92a can potentially bind to the DAB2IP $3^{\prime} \mathrm{UTR}$ to regulate the protein expression of DAB2IP. Based on miRNA database analysis, we found a potential miR92a binding site on the DAB2IP 3'UTR (Fig. 4a). We constructed (length $=2 \mathrm{~kb}$ ) a wild-type (WT) $3^{\prime}$ UTR of DAB2IP on the $3^{\prime}$ end of luciferase cDNA (psiCheck2-DAB2IP $3^{\prime} \mathrm{UTR}-\mathrm{WT}$ ), and the luciferase reporter assay showed that miR-92a could decrease the luciferase activity of psiCheck2-DAB2IP $3^{\prime}$ UTR-WT (Fig. 4b, lane 3 vs. 1). To determine whether this binding of miR-92a to the DAB2IP 3'UTR is specific, we also constructed a mutant $3^{\prime} \mathrm{UTR}$ of DAB2IP, with a mutation that destroys the miR92a binding site (psiCheck2-DAB2IP 3' UTR-mt). The luciferase activity assay results showed that this mutation could prevent the binding of miR-92a (Fig. 4b, lane 4 vs. 2).

\section{Mechanism dissection: How ER $\beta$ can modulate miR-92a at the transcriptional level}

Because the mature form of miR-92a was derived from its host gene C13orf25 (Fig. 4c), we also tested whether ER $\beta$ could transcriptionally regulate the expression of C13orf25. Real-time quantitative-PCR showed that enforced expression of ER $\beta$ could up-regulate C13orf25 and that knockdown of ER $\beta$ could down-regulate C13orf25 at the mRNA levels (Fig. 4d).

To investigate whether ER $\beta$ could regulate C13orf25 by transcriptional regulation of its promoter activity, we analyzed the potential ER $\beta$-response-elements (EREs) on the $5^{\prime}$ promoter of the C13orf25 gene. The bioinformatics analysis showed four potential EREs in the $4 \mathrm{~kb}$ promoter region (Fig. 4c), namely, locations (\#1 and \#2) -3963 to $-3887 \mathrm{bp}$, (\#3) -3045 to $-3035 \mathrm{bp}$, and (\#4) -2398 to $-2390 \mathrm{bp}$. To test the binding of ER $\beta$ to these potential EREs, the chromosome immuno-precipitation assay (ChIP) was applied, and the results showed that ER $\beta$ could selectively bind to one of the candidate ERE sites, i.e., the predicted \#4 ERE, located from the -2398 base to the -2390 base (Fig. 4e). We cloned $2.4 \mathrm{~kb}$ of the promoter region into the pGL3 vector for the luciferase reporter assay. The results showed that ER $\beta$ could increase the C13orf $25(2.4 \mathrm{~kb})$ promoterluciferase reporter (2.4 kb) activity (Fig. 4f). 

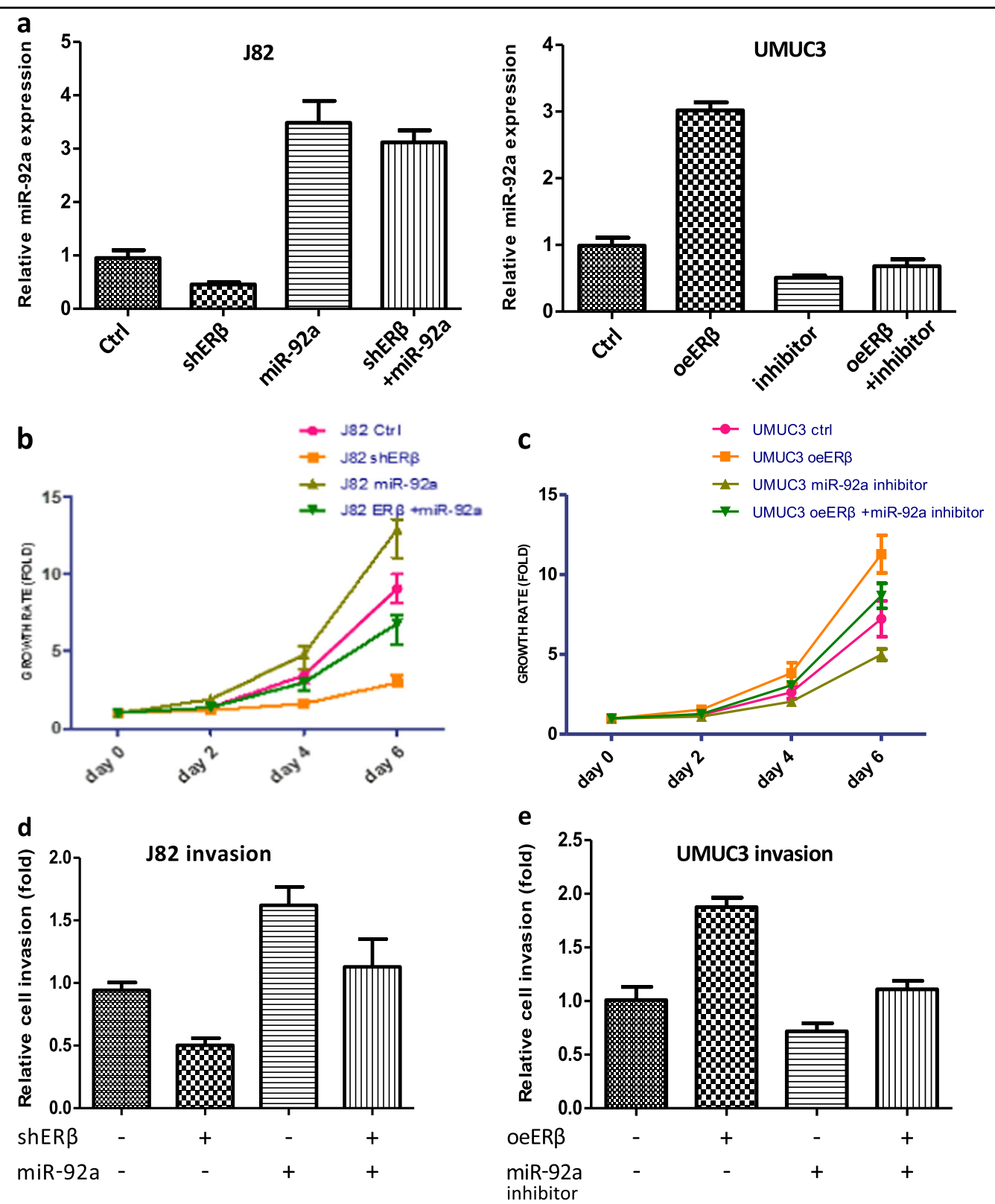

Fig. 2 The miR-92a is an oncogenic miRNA that plays critical roles in mediating ERß-promoted BCa cell growth and invasion. a Real-time PCR shows miR-92a levels in J82 cells transduced with lentiviral ERß shRNA (shERß) with/without miR-92a or control (Ctrl) and in UMUC3 cells overexpressed with lentiviral ERß CDNA (oeERß) with/without miR-92a inhibitor. We first showed in the J82 cells (left) that shERß can reduce miR-92a expression. In UMUC3 cells (right), when ERß is overexpressed, the miR-92a was up-regulated. The transfection of miR-92a inhibitor (oligonucleotide) can significantly reduce ER $\beta$-upregulated miR-92 in UMUC3 cells. b J82 cells were prepared as in A for MTT assays. Ectopic miR-92a expression could reverse the shER $\beta$-reduced BCa growth in J82 cells. Analysis of cell growth was conducted by MTT assay on days 0, 2, 4 and 6. c UMUC3 cells were prepared as described in A. The miRNA-92a inhibitor could dramatically reduce ERß-promoted cell growth in these UMUC3 cells. $\mathbf{d}$ Ectopic miR-92a expression could reverse shERß-reduced BCa invasion in $J 82$ cells. Invasion assays were conducted using the transwell Matrigel invasion assay. e Transfection of UMUC3 cells with miR-92a inhibitor can reduce ER $\beta$-promoted cell invasion. Data are represented as the mean \pm SD

Preclinical study using an in vivo mouse BCa model to demonstrate that $\mathrm{ER} \beta$ increases $\mathrm{BCa}$ metastasis via alteration of miR-92a/DAB2IP signals

To confirm the in vitro results in an in vivo mouse $\mathrm{BCa}$ model, we used J82 cells orthotopically xenografted into the bladder walls of female nude mice. The implanted J82 cells were stably transfected with pCDNA3-luciferase (J82-Luc) for monitoring of the tumor growth and metastasis via the real-time in vivo imaging system (IVIS). The J82-Luc cells were transduced with lentiviral vector control, shER $\beta$, miR-92a, or $\operatorname{shER} \beta+\operatorname{miR}-92 \mathrm{a}$, and $1 \times$ $10^{6}$ of each type of cells were implanted into the bladder 


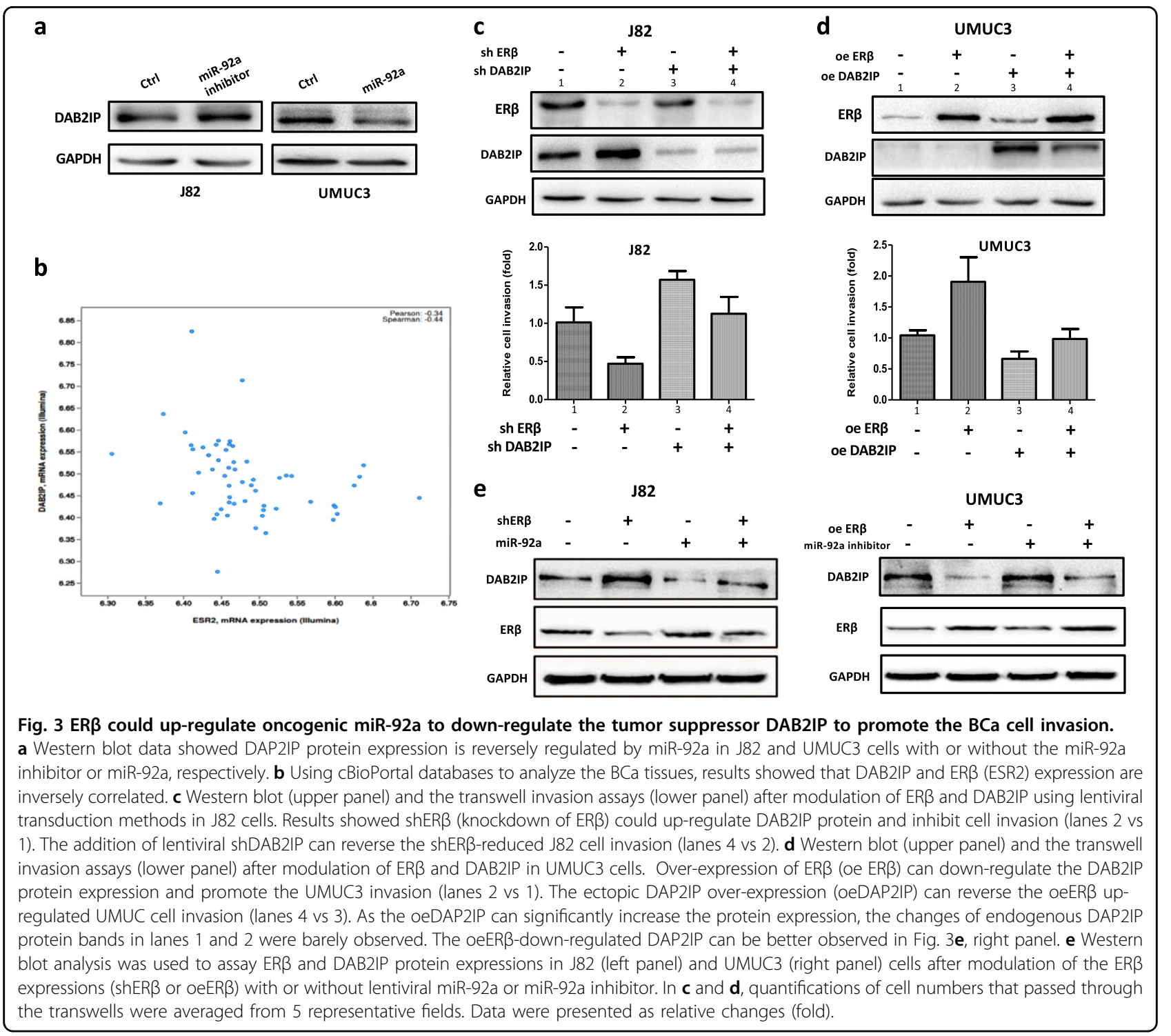

wall of 6-week-old female nude mice $(n=8) .5$ weeks after tumor implantation, we applied IVIS to monitor tumor development weekly for an additional 3 weeks.

The mice in all four groups developed tumors with different growth and metastasis rates. The IVIS imaging results revealed that the mice implanted with J82-Luc cells with miR-92a overexpression had the highest metastatic incidence (75\%). In contrast, the J82-luc shER $\beta$ tumor group had the lowest metastatic incidence (12.5\%). Comparison of female mice with J82-luc $+\operatorname{shER} \beta$ vs. those with J82-luc + shER $\beta+$ miR-92a xenografts showed that miR-92a can reverse the shER $\beta$-reduced metastatic foci formation (Fig. 5a, b).

After the mice were euthanized, we examined the weights of bladders with tumors and the total metastatic foci. The average bladder weight of the J82 shER $\beta$ group was significantly lower than that of the control group, and the average bladder weight of the miR-92a group was higher than that of the control group (Fig. 5c). The results revealed that the metastatic foci were located in the retroperitoneum, peritoneal, and diaphragm regions (Fig. 5d). The results from IHC staining in Fig. 5e also indicated the correlated expressional changes of DAB2IP by $E R \beta$ in the bladder tumors, which were consistent with the in vitro data. We used qRT-PCR to examine the expression levels of ER $\beta$ mRNA, miRNA-92a and DAB2IP mRNA in the metastatic foci collected from peritoneal regions. As shown in the Supplementary Figure 1: The miRNA-92a was up-regulated in the metastatic foci in J82 miRNA-92a group compared to other groups and the miRNA-92a was down-regulated in J82 shER $\beta$ group. The DAB2IP mRNA did not significantly change among those four tumor groups which implied that DAB2IP may be regulated by miRNA-92a through post- 


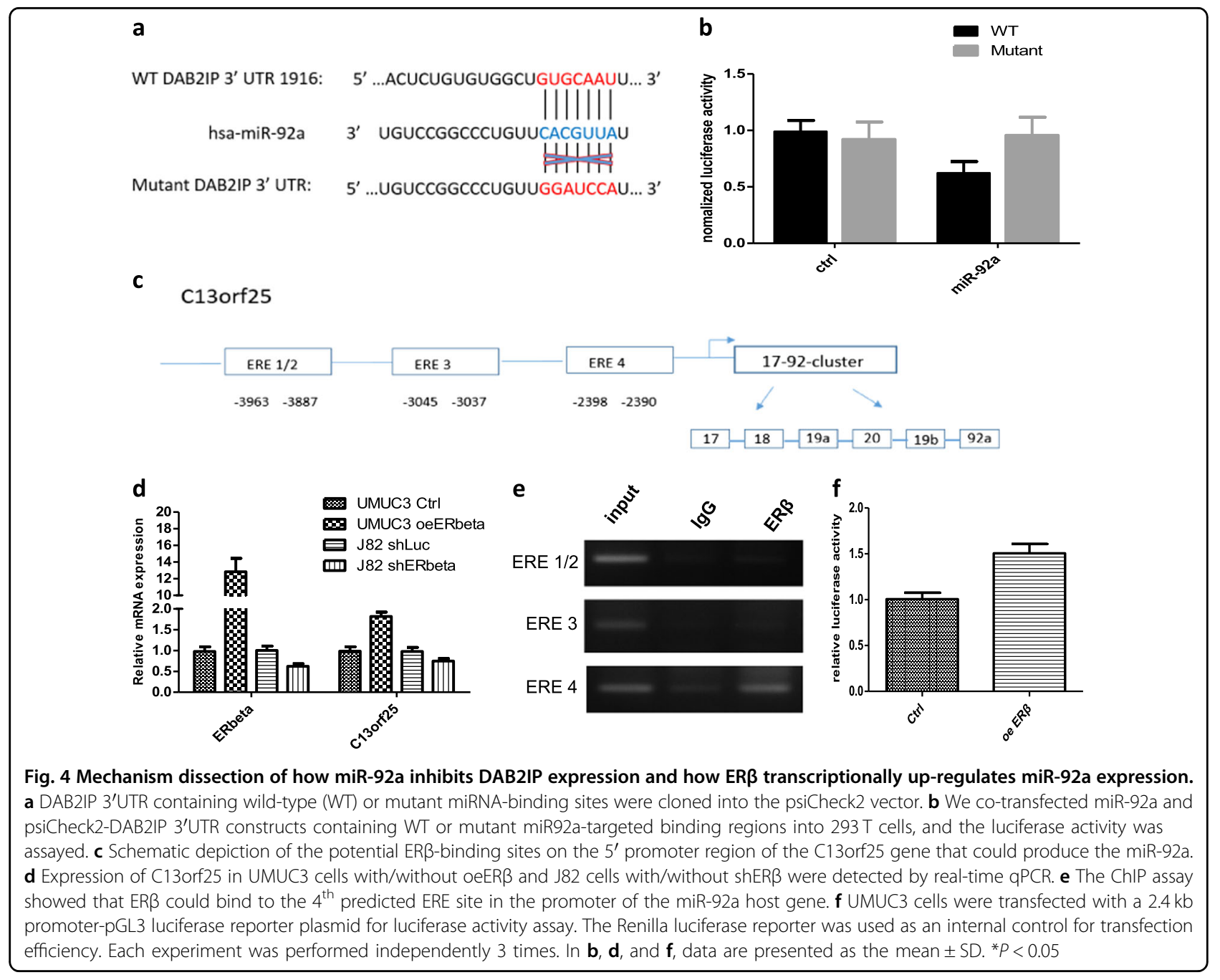

transcriptional control. These in vivo findings are consistent with the in vitro results. Together, our results demonstrated that ER $\beta$ could increase $B C$ a growth and metastasis via miR-92a/DAB2IP signals in the orthotopically implanted mouse BCa model.

\section{Discussion}

Clinical data show that sexual disparity exists in $\mathrm{BCa}$ incidence and progression. Men are more likely to get $\mathrm{BCa}$, and in contrast, women have a higher muscleinvasive $\mathrm{BCa}$ rate than men. ERs might be responsible for this sexual difference. In the context of $\mathrm{BCa}$, the two receptors ER $\alpha$ and ER $\beta$ have been studied ${ }^{3,22,23}$. Observations in clinical samples implicated that ER $\beta$ expression is positively linked to cancer stage ${ }^{23}$. Our previous studies using ER knockout mouse models showed that ER $\alpha$ can inhibit $\mathrm{BCa}$ initiation and growth ${ }^{4}$ and that $\mathrm{ER} \beta$ can promote $\mathrm{BCa}$ growth and invasion ${ }^{5}$. We applied a BBNinduced mouse $\mathrm{BCa}$ model to demonstrate that knockout of ER $\beta$ can decrease $\mathrm{BCa}$ occurrence significantly ${ }^{5}$.
Although our previous study showed that ER $\beta$ could function via up-regulation of MCM $5^{5} \mathrm{CCL} 2^{24}$, and IL-1/ c-Met ${ }^{25}$ pathways to promote the $\mathrm{BCa}$, the mechanisms by which $E R \beta$ can increase $B C$ a growth and metastasis are not yet fully investigated.

As an important player in promoting $\mathrm{BCa}$ progression, ER $\beta$ could have multiple ways to exert its function. We focused on miRNAs because they are involved in the progression of $\mathrm{BCa}^{26,27}$. In this work, we screened 30 miRNAs related to $\mathrm{BCa}$ progression ${ }^{26,28}$ and found that a subset of them are regulated by ER $\beta$. Interestingly, we found miR92a, which is located in a primary transcript known as C13orf25 and belongs to the miR-17-92 cluster $^{29}$, is a critical downstream factor of ER $\beta$. This miRNA has been reported to enhance the progression of different cancers, including lung cancer, hepatocellular carcinoma, colorectal cancer and neuroblastoma ${ }^{30,31}$. Thus far, several targeted genes of miR-92a have been identified, such as the Bcl-2interacting mediator of cell death (BIM) and reversioninducing cysteine-rich protein with Kazal motifs (RECK), 
a

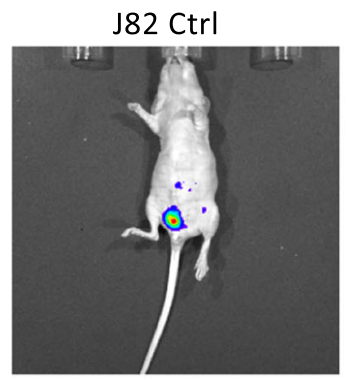

b

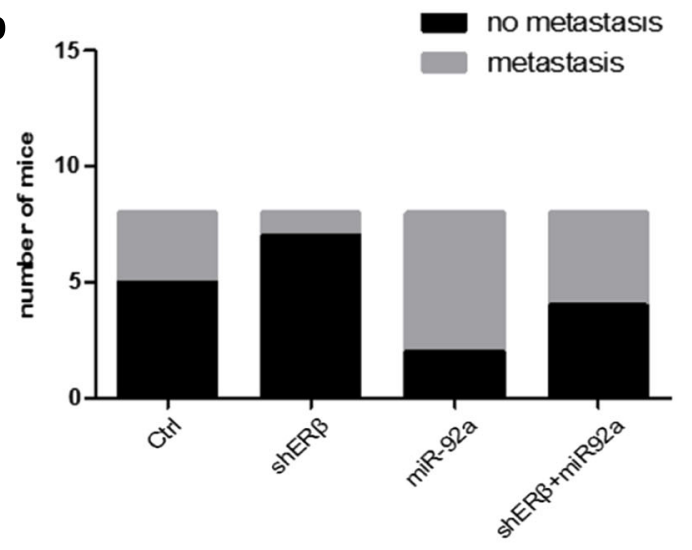

d

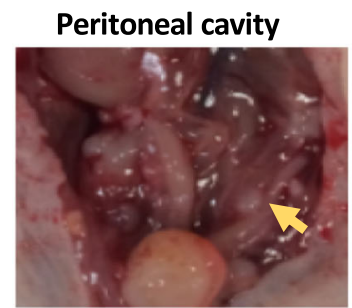

e

J82 Ctrl
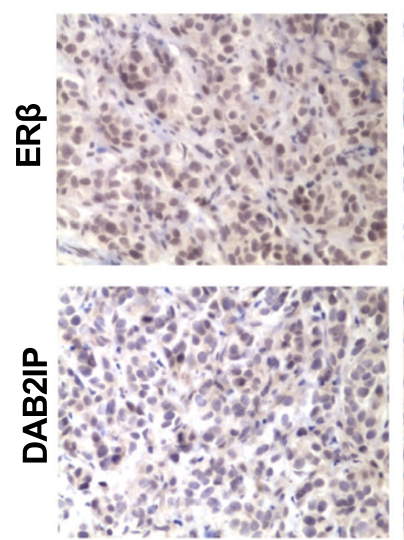

J82 shER $\beta$

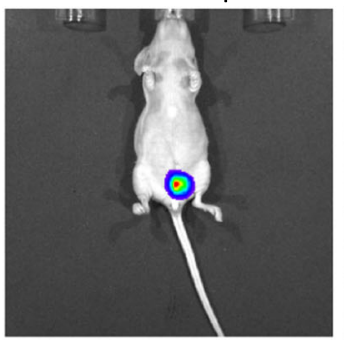

no metastasıs
J82 ShER $\beta$
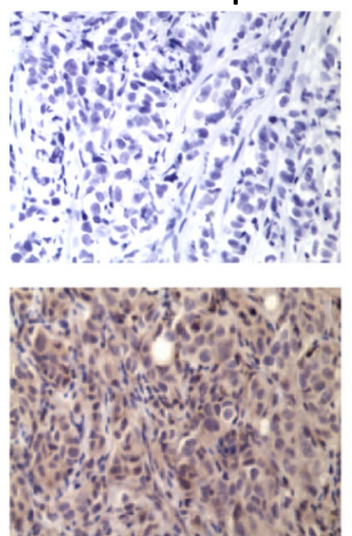
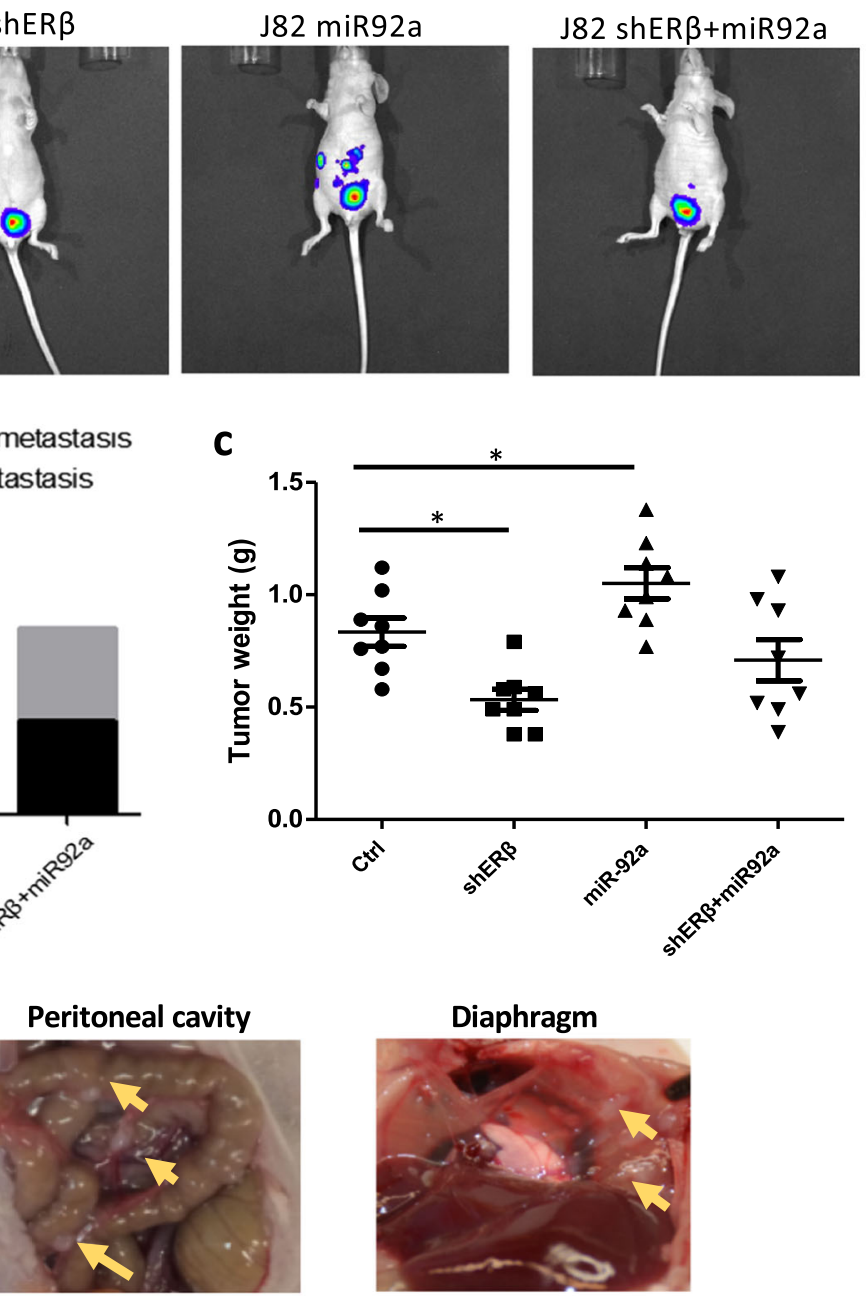

Diaphragm

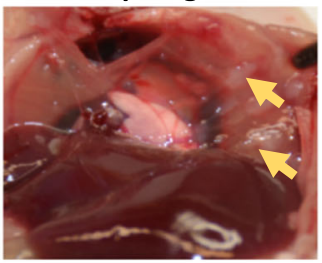

J82 shER $\beta+$ miR92a

\section{J82 miR92a}
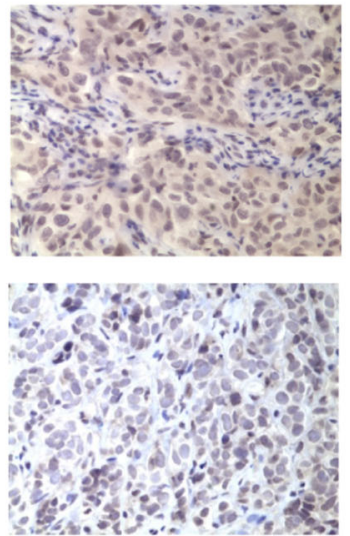
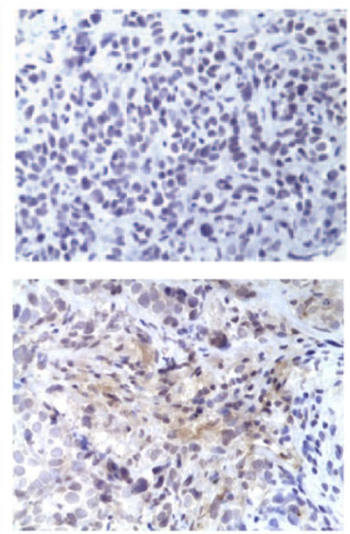

Fig. 5 ER $\beta$ promotes metastasis of $B C a$ via up-regulation of miR-92a and down-regulation of DAB2IP in the in vivo mouse $B C a$ model. The implanted J82 cells were stably transfected with PCDNA3-luciferase (J82-Luc) for monitoring of tumor growth and metastasis via the real-time in vivo imaging system (IVIS). a Representative IVIS images of the four groups of mice showed the outcome at eight weeks after orthotopical implantation of BCa J82-luc cells into the bladder wall of female nude mice. $\mathbf{b}$ Incidence of metastases in different groups of mice at eight weeks after BCa cell implantation. c Comparison of bladder weights of different mouse tumor groups. $\mathbf{d}$ The images show representative metastatic lymph foci (yellow arrows) around the peritoneal and diaphragm regions. e Representative images of IHC results for ER $\beta$ and DAB2IP in four groups of orthotopic bladder tumor tissues $(400 \times)$. In $\mathbf{c}$, data are presented as the mean $\pm S D,{ }^{*} P<0.05$ 
which are all tumor suppressors ${ }^{30}$. In the current study, overexpression of miR-92a could significantly increase $\mathrm{BCa}$ cell growth and invasion. We supply the first evidence that miR-92a expression is regulated by ER $\beta$, and exhibits oncogenic activity in BCa. Importantly, interruption of miR92a can partially block ER $\beta$ 's function in BCa progression.

We also identified DAB2IP as a new in vivo target of miR92a. DAB2IP is involved in the development of many cancers. By interacting with DIP1/2, a Ras GTPase-activating protein, DAB2IP can form a protein complex with a negative regulatory activity that modulates the Rasmediated signaling pathway ${ }^{32}$. As a tumor suppressor, DAB2IP was found to be down-regulated in BCa tissues. Clinical sample analysis and functional experiments revealed that DAB2IP plays an important role in BCa progression and was strongly correlated with unfavorable patient outcomes ${ }^{16}$. Loss of DAB2IP can also influence the response of $\mathrm{BCa}$ cells to ionizing radiation ${ }^{33}$. In our study, DAB2IP was found to be down-regulated in BCa cells with ER $\beta$ overexpression and up-regulated in $\mathrm{BCa}$ cells with knockdown of endogenous ER $\beta$ (Fig. 2). Consistent results were obtained in an orthotopically implanted mouse $\mathrm{BCa}$ model (Fig. 5). Ectopic expression of DAB2IP can partially reverse ER $\beta$ overexpression-mediated $B C a$ cell growth and invasion, suggesting that DAB2IP indeed contributes to $E R \beta$ 's function in $\mathrm{BCa}$. The $\mathrm{IHC}$ results of the in vivo mouse $\mathrm{BCa}$ tissues also showed that ER $\beta$ could down-regulate DAB2IP expression (Fig. 5e).

By analyzing the cBioPortal databases, we found an inverse correlation exists between the expressions of ER $\beta$ and DAB2IP. These clinical data strongly support our cell line findings. In the current study, we found a new ER $\beta$ downstream miR-92a/DAB2IP signaling pathway that is critical for the ER $\beta$ function of promoting BCa progression. The control of growth and invasion of $\mathrm{BCa}$ cells by the ER $\beta / \mathrm{miR}-92 \mathrm{a} / \mathrm{DAB} 2 \mathrm{IP}$ axis might offer new strategies for treatment of $\mathrm{BCa}$. Future studies might allow the development of an effective therapeutic strategy to interrupt these newly identified signals and better suppress $\mathrm{BCa}$ progression.

\section{Acknowledgements}

The authors thank Dr. J. T. Hsieh (UT Southwestern, TX) for supplying the retroviral DAB2IP plasmid and Karen Wolf for assistance with manuscript preparation. Zhenyu Ou was supported by the National Natural Science Foundation of China (81702531).

\footnotetext{
Author details

'Departments of Urology and Plastic Surgery, Xiangya Hospital, Central South University, Changsha 410008, China. ${ }^{2}$ Departments of Urology and Pathology, University of Rochester Medical Center, Rochester, New York 14642, USA. ${ }^{3}$ Department of Urology, Changzhou No. 2 People's Hospital Affiliated to Nanjing Medical University, Changzhou 213003, China. ${ }^{4}$ Departments of Medicine, University of Rochester Medical Center, Rochester, New York 14642, USA
}

Conflict of interest

The authors declare that they have no conflict of interest.

\section{Publisher's note}

Springer Nature remains neutral with regard to jurisdictional claims in published maps and institutional affiliations.

Supplementary information accompanies this paper at https://doi.org/ 10.1038/s12276-018-0155-5.

Received: 22 December 2017 Revised: 7 May 2018 Accepted: 29 May 2018. Published online: 20 November 2018

\section{References}

1. Sievert, K. D. et al. Economic aspects of bladder cancer: what are the benefits and costs? World J. Urol. 27, 295-300 (2009).

2. Siegel, R. L., Miller, K. D. \& Jemal, A. Cancer statistics, 2018. CA Cancer J. Clin. 68, 7-30 (2018).

3. Hsu, l., Vitkus, S., Da, J. \& Yeh, S. Role of oestrogen receptors in bladder cancer development. Nat. Rev. Urol. 10, 317-326 (2013).

4. Hsu, I. et al. Estrogen receptor alpha prevents bladder cancer via INPP4B inhibited akt pathway in vitro and in vivo. Oncotarget 5, 7917-7935 (2014).

5. Hsu, I. et al. Suppression of ERbeta signaling via ERbeta knockout or antagonist protects against bladder cancer development. Carcinogenesis 35, 651-661 (2014).

6. Yang H., Fang F., Chang R., Yang L. MicroRNA-140-5p suppresses tumor growth and metastasis by targeting TGFBR1 and FGF9 in hepatocellular carcinoma. Hepatology. 58, 205-217 (2013).

7. Liu X., et al. Regulation of microRNAs by epigenetics and their interplay involved in cancer. J. Exp. Clin. Cancer Res. 32, 96 (2013).

8. Zhang S., et al. MicroRNA-24 upregulation inhibits proliferation, metastasis and induces apoptosis in bladder cancer cells by targeting CARMA3. Int. J. Oncol. 47,1351-1360 (2015).

9. Barbato, S., Solaini, G. \& Fabbri, M. MicroRNAs in oncogenesis and tumor suppression. Int. Rev. Cell. Mol. Biol. 333, 229-268 (2017).

10. Paris, O. et al. Direct regulation of microRNA biogenesis and expression by estrogen receptor beta in hormone-responsive breast cancer. Oncogene 31, 4196-4206 (2012).

11. Zhou, J. et al. Loss of DAB2IP in RCC cells enhances their growth and resistance to mTOR-targeted therapies. Oncogene 35, 4663-4674 (2016).

12. Liao, H. et al. microRNA-32 induces radioresistance by targeting DAB2IP and regulating autophagy in prostate cancer cells. Oncol. Lett. 10, 2055-2062 (2015).

13. Liu, L., Xu, C., Hsieh, J. T., Gong, J. \& Xie, D. DAB2IP in cancer. Oncotarget $\mathbf{7}$, 3766-3776 (2016)

14. Zhang, X. et al. Low expression of DAB2IP contributes to malignant development and poor prognosis in hepatocellular carcinoma. J. Gastroenterol. Hepatol. 27, 1117-1125 (2012).

15. $\mathrm{Wu}, \mathrm{K}$ et al. DAB2IP regulates the chemoresistance to pirarubicin and tumor recurrence of non-muscle invasive bladder cancer through STAT3/Twist1/P-glycoprotein signaling. Cell. Signal. 27, 2515-2523 (2015).

16. Shen, Y. J. et al. Downregulation of DAB2IP results in cell proliferation and invasion and contributes to unfavorable outcomes in bladder cancer. Cancer Sci. 105, 704-712 (2014).

17. Ou, Z. et al. Tumor microenvironment B cells increase bladder cancer metastasis via modulation of the IL-8/androgen receptor (AR)/MMPs signals. Oncotarget 6, 26065-26078 (2015).

18. Yoshino, H. et al. Aberrant expression of microRNAs in bladder cancer. Nat. Rev. Urol. 10, 396-404 (2013)

19. Ghildiyal M, Zamore PD. Small silencing RNAs: an expanding universe. Nat. Rev. Genet. 10, 94-108 (2009).

20. Vlachos, I. S. et al. DIANA-TarBasev7.0: indexing more than half a million experimentally supported miRNA:mRNA interactions. Nucleic Acids Res. 43, D153-D159 (2015). 
21. Gao, J. et al. Integrative analysis of complex cancer genomics and clinical profiles using the cBioPortal. Sci. Signal. 6, pl1 (2013).

22. Sonpavde, G. et al. Efficacy of selective estrogen receptor modulators in nude mice bearing human transitional cell carcinoma. Urology 69, 1221-1226 (2007).

23. Miyamoto, $\mathrm{H}$. et al. Expression of androgen and oestrogen receptors and its prognostic significance in urothelial neoplasm of the urinary bladder. BJU Int. 109, 1716-1726 (2012).

24. Rao Q. et al. Recruited mast cells in the tumor microenvironment enhance bladder cancer metastasis via modulation of ERß/CCL2/CCR2 EMT/MMP9 signals. Oncotarget. 7, 7842-7855 (2016).

25. Tao L. et al. Recruited $T$ cells promote the bladder cancer metastasis via up-regulation of the estrogen receptor $\beta / \mathrm{LL}-1 / \mathrm{C}-\mathrm{MET}$ signals. Cancer Lett. 430, 215-223(2018)

26. Catto, J. W. et al. Distinct microRNA alterations characterize high- and lowgrade bladder cancer. Cancer Res. 69, 8472-8481 (2009).

27. Xiong $Y$. et al. The Long Non-Coding RNA XIST Interacted with MiR124 to Modulate Bladder Cancer Growth, Invasion and Migration by Targeting Androgen Receptor (AR). Cell Physiol Biochem. 43, 405-418 (2017).
28. Guancial, E. A., Bellmunt, J., Yeh, S., Rosenberg, J. E. \& Berman, D. M. The evolving understanding of microRNA in bladder cancer. Urol. Oncol. 32, 41 e31-e40 (2014)

29. Ota, A. et al. Identification and characterization of a novel gene, C13orf25, as a target for 13q31-q32 amplification in malignant lymphoma. Cancer Res. 64, 3087-3095 (2004)

30. Lin, H. Y., Chiang, C. H. \& Hung, W. C. STAT3 upregulates miR-92a to inhibit RECK expression and to promote invasiveness of lung cancer cells. Br. J. Cancer 109, 731-738 (2013).

31. Shigoka, M. et al. Deregulation of miR-92a expression is implicated in hepatocellular carcinoma development. Pathol. Int. 60, 351-357 (2010).

32. Chen, H., Tu, S. W. \& Hsieh, J. T. Down-regulation of human DAB2IP gene expression mediated by polycomb Ezh2 complex and histone deacetylase in prostate cancer. J. Biol. Chem. 280, 22437-22444 (2005).

33. Zhang, T., Shen, Y., Chen, Y., Hsieh, J. T. \& Kong, Z. The ATM inhibitor KU55933 sensitizes radioresistant bladder cancer cells with DAB2IP gene defect. Int. J. Radiat. Biol. 91, 368-378 (2015). 